\title{
Imaging of Internal Defects of Polymer-Modified Wood Using Total Focusing Method
}

\author{
Liping Sun, ${ }^{1}$ Hongju Zhou, ${ }^{1}$ Hongwei Zhou $\mathbb{D}^{1},{ }^{1}$ Guizhong Jiao, ${ }^{2}$ and Ling $\mathrm{Ma}^{1}$ \\ ${ }^{1}$ Northeast Forestry University, Harbin, Heilongjiang, China \\ ${ }^{2}$ East China Optoelectronic Integrated Device Research Institute, Bengbu, Anhui, China \\ Correspondence should be addressed to Hongwei Zhou; easyid@163.com
}

Received 18 April 2019; Accepted 29 May 2019; Published 17 June 2019

Guest Editor: Changlei Xia

Copyright (c) 2019 Liping Sun et al. This is an open access article distributed under the Creative Commons Attribution License, which permits unrestricted use, distribution, and reproduction in any medium, provided the original work is properly cited.

\begin{abstract}
Polymer modification can improve the stability and corrosion resistance of wood, but it could create defects inside wood during the modification processing. Detection of defects inside polymer-modified wood can reduce wood losses and prevent the occurring of defects. Data simulation and tomographic imaging of polymer-modified wood internal defects were carried out using electromagnetic waves with nondestructive testing. This study constructed the polymer-modified wood models, simulated the electromagnetic scattering wave, and used the total focusing method to perform tomography of the defects in the polymermodified wood. By analyzing the imaging characteristics of different types of defects, the effectiveness of electromagnetic waves in the detection of internal defects of polymer-modified wood was proved. This method can be extended to test internal defects of other high molecular polymers.
\end{abstract}

\section{Introduction}

As a renewable material, wood has been widely used in various fields such as construction, decoration, and energy. However, defects of wood such as warp, crack, and decay caused by the changing of external temperature and humidity have caused serious economic losses in wood production [1]. In order to reduce the defects of wood and improve its physical and chemical properties, the modification of wood began in the early 20 th century $[2,3]$. The stability and corrosion resistance of polymer-modified wood have been significantly improved compared to the wood modified by other modification methods and gradually became the main method of wood modification $[4,5]$. The polymer-modified wood has a wide range of applications in buildings and outdoor wood products. However, defects may occur inside wood during processing or service, and the detection of defects inside the polymer-modified wood can reduce losses and prevent from the defect occurring in advance [6].

The nondestructive testing technology can find the defects of wood by comparing the differences of physical or chemical characteristics [7]. Methods such as the electromagnetic wave detection, laser detection, infrared detection, ultrasonic detection, nuclear magnetic resonance, and X-ray detection have been widely used in the metal defect detection, ground penetrating radar, medical imaging, and other fields [8-10]. There are various methods for detecting polymer-modified wood, such as the infrared detection, ultrasonic detection, stress wave detection, and X-ray detection [11-13]. Each of these methods has its advantages and disadvantages, but there is no single method that can easily and effectively detect the internal defects of the polymer-modified wood. Infrared detection method detects the internal defects of polymer-modified wood by the phenomenon of abnormal temperature rise, but it is greatly affected by environmental factors. Sunlight, airflow, air temperature and humidity, and surface leakage current would affect the detection signals of the infrared detection method [14]. The ultrasonic detection method requires coupling agent and needs to select a probe that matches the acoustic impedance which requires close contact with the surface of the testing samples, which limits its application range [15]. The stress wave detection requires the sensors to be nailed to the polymer-modified wood, which would cause damage. The X-ray inspection equipment 
costs too much and also has a safety concern; e.g., if used improperly, it can cause radiant damage to human body [16].

In this paper, the total focusing method was applied to make the image inversion of the electromagnetic wave propagation data in the composite insulator to achieve the purpose of nondestructive testing. The total focus method is mostly used in phased array ultrasonic monitoring systems; this method has been widely used in many fields such as petroleum exploration and industrial product testing $[17,18]$. The principle is to coherently sum all the elements to focus at each point in the image reconstruction area. This method can define various reconstruction networks and achieve highprecision reconstruction $[19,20]$.

\section{Method}

The total focusing method collects data by the full matrix capture method, and the acquisition step is to record the signals between all array elements as shown in Figure 1. The signals collected by the transmitting element $i$ and the receiving element $j$ are denoted by $E\left(t, u_{i}, v_{j}\right)$, where $u_{i}$ and $v_{j}$, respectively, represent the positions of the transmitting array elements and the receiving array elements. If $N$ represents the total number of elements, the data set is defined as $E(t, u, v)(1 \leq i \leq N, 1 \leq j \leq N)$. As for reconstruction of any sample point $(x, y)$ in the solution domain, it can be given by the following.

$$
O(x, y)=\sum_{i=1}^{N} \sum_{j=1}^{N} E\left(\frac{r_{1}+r_{2}}{c / \sqrt{\varepsilon}}+\tau, u_{i}, v_{j}\right)
$$

In the formula $r_{1}=\sqrt{\left(x-u_{i}\right)^{2}+y^{2}}, r_{2}=\sqrt{\left(x-v_{j}\right)^{2}+y^{2}}, \mathrm{c}$ is the propagation speed of electromagnetic waves in vacuum, $\varepsilon$ is the relative dielectric constant of the background medium (assuming that the polymer-modified wood material is uniform and isotropic) [21, 22], and $\tau$ is the time constant. The calculation process is equivalent to focus at each point $(x, y)$.

The time constant $\tau$ consists of two parts: one is from the gprMax simulation software, and the other depends on the wavelength of the excited electromagnetic wave. There is a "zero zone" of the initial emission time in the gprMax simulation software, and no electromagnetic waves are emitted in this region. That is, the electromagnetic wave starts to emit later than zero-time. In the imaging result, the data of each sampling point comes from the value at the peak of the Ricker wave, which corresponds to $\lambda / 2 \mathrm{c}$. The time constant $\tau$ corresponding to the electromagnetic wave of $1 \mathrm{GHz}$ is $1.6121 \mathrm{~ns}$.

A certain transmitting point of the electromagnetic wave array element transmits a signal which is received by all other receiving points, which means that "one transmits and more receive" the electromagnetic wave signal. This process is repeated for all array elements, which means "point-by-point transmission, total focus".

Acrylonitrile is a good wood intumescent agent, which can be used for impregnating the plasticized wood to increase its dimensional stability. In this paper, the acrylonitrile modified wood was taken as an example to develop a defect

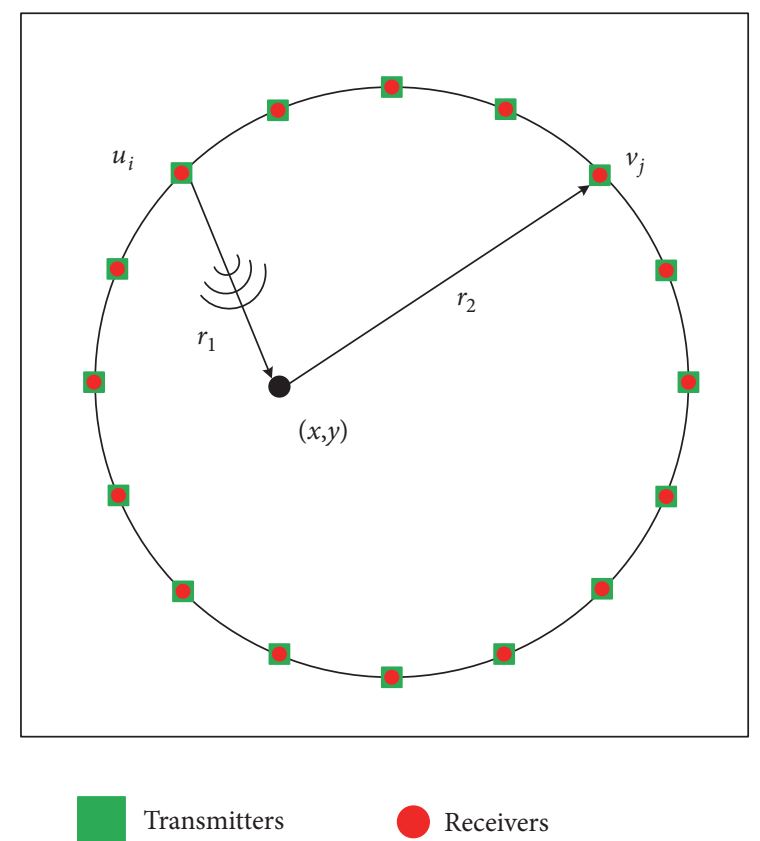

FIGURE 1: Schematic diagram of data acquisition for the total focusing method.

simulation model, and its factors were set to be a shape diameter of $0.18 \mathrm{~m}$, a relative dielectric constant of 8 , the dielectric loss tangent of 0.0038 , the volume resistivity of $8.93 \times 10^{8} \mathrm{M} \cdot \Omega \cdot \mathrm{cm}$, the surface resistivity of $9.287 \times$ $10^{8} \mathrm{M} \cdot \Omega$, and the heat distortion temperature of $307^{\circ} \mathrm{C}$. A two-dimensional space model of the solution domain $(0.2 \mathrm{~m}$, $0.2 \mathrm{~m}$ ) was developed using the gprMax simulation software, on a circumference with a center of $(0.1 \mathrm{~m}, 0.1 \mathrm{~m})$ and a radius of $0.09 \mathrm{~m}, N=16$ array elements, which were equally spaced and each array element had a transmitter and a receiver. The transmitter sequentially emits a Ricker wave with a frequency of $f=1 \mathrm{GHz}$, and the waveform function is $\chi(t)=$ $-A\left[2 \pi^{2} f^{2}(t-\sqrt{2} / f)^{2}-1\right] e^{-\pi^{2} f^{2}(t-\sqrt{2} / f)^{2}}, t>0$, received by all receivers. Time window $t_{w}=2 \mathrm{y} /(\mathrm{c} / \sqrt{\varepsilon})+1 / f+\tau \approx 9$ ns.

\section{Experimental Results and Analysis}

In this study, the circular polymer-modified wood model with preset defects was taken as the research sample. The electromagnetic wave was used to simulate the nondestructive testing of polymer-modified wood. The position, size, shape, and other related information of the defects were obtained by the electromagnetic wave propagating in the medium. Specifically, it involved the influence of the type, position, shape, and quantity of the defect medium on the inversion imaging of the electromagnetic wave total focusing method.

3.1. Single Circular Air Defect Simulation Imaging. The internal defects of the polymer-modified wood were set to be a circular air diameter of $0.02 \mathrm{~m}$, and the circle center at $(0.1 \mathrm{~m}$, 0.1m). As shown in Figure 2, the model was simulated using 


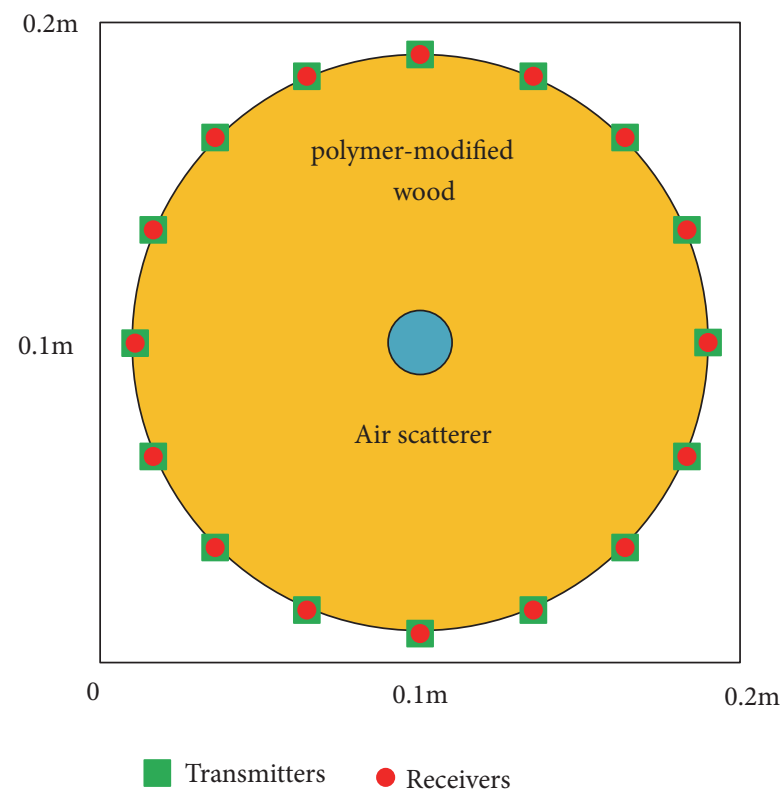

FIgURE 2: Single circular air defect model diagram.

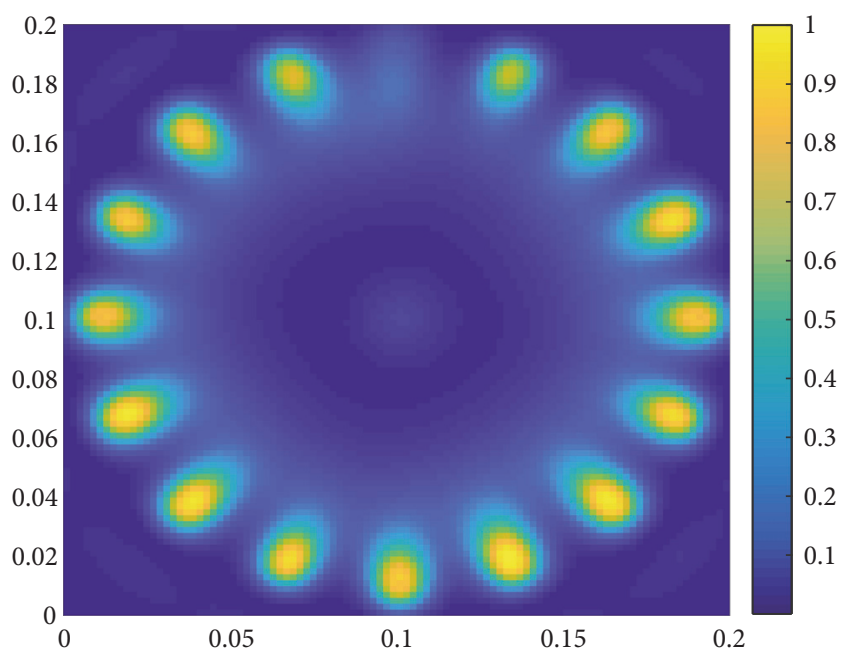

FIgURE 3: Single circular air defect inversion imaging diagram.

the gprMax software. The inversion results are shown in Figure 3. As shown in the figure, the air defect (weak scattered) was insensitive to the scattering of electromagnetic waves, there were obvious bright spots at the 16 array elements, and the defect position information was not obvious.

\subsection{Single Circular Metal Defect Simulation Imaging. In order} to explore the problem that the air defect imaging was not obvious, the defect was set to a circular metal with a diameter of $0.02 \mathrm{~m}$, and the circle center at $(0.1 \mathrm{~m}, 0.1 \mathrm{~m})$. Figure 4 shows the model that was simulated by using gprMax software. The inversion results are shown in Figure 5. As shown in Figure 5, the metal defects (strong scatterers) were sensitive to electromagnetic waves, and the total focusing method can clearly and accurately image the metal defects.

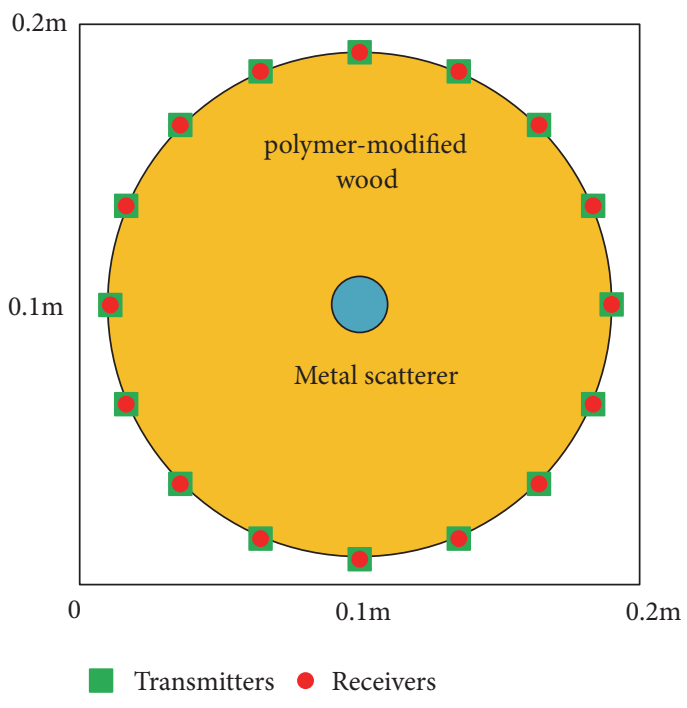

Figure 4: Single circular metal defect model.

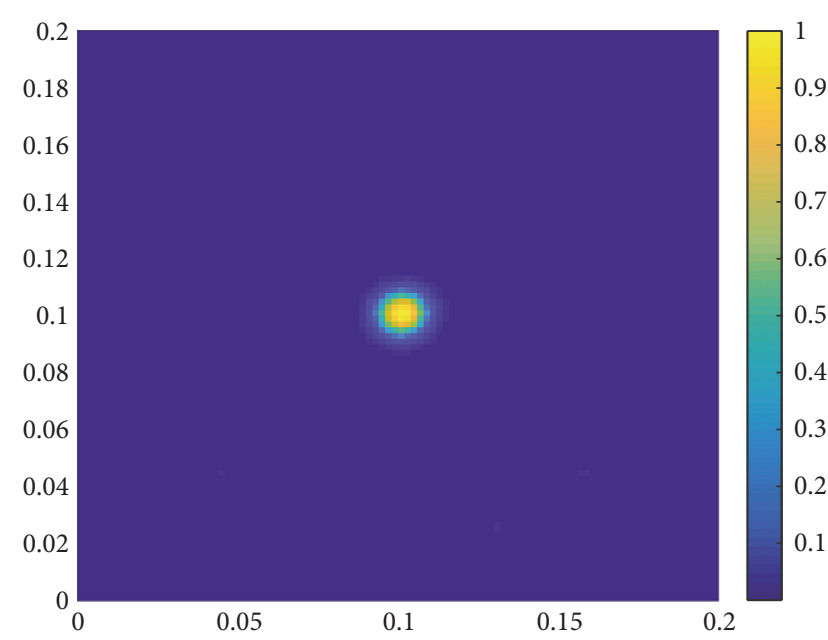

FIGURE 5: Single circular metal defect inversion image.

3.3. Improvement of the Total Focusing Method of Weak Scatterers. By comparing the A-scan data of the metal defect model, the air defect model and the no defect model at the same array are shown in Figure 6. It was found that the curves of the metal defect model and the air defect model were similar to those of the defect-free model in the range of 0 to $2.57 \mathrm{~ns}$, where the data came from the electromagnetic wave signals emitted by the transmitter at the same array element, which was not directly received by the receiver through the scatterer. In the range of $2.57 \mathrm{~ns}$ to $4 \mathrm{~ns}$, the curve of the metal defect model was significantly different from that of the defect-free model, and the difference between the air defect model and the defect-free model was small, where the data was from the scattering field data of the electromagnetic wave in the medium propagation process. In the range of $4 \mathrm{~ns}$ to $8 \mathrm{~ns}$, the curves of the three models were similar, and the data fluctuation amplitude was much smaller than the data fluctuation in the range of 0 to $4 \mathrm{~ns}$, where the data came 


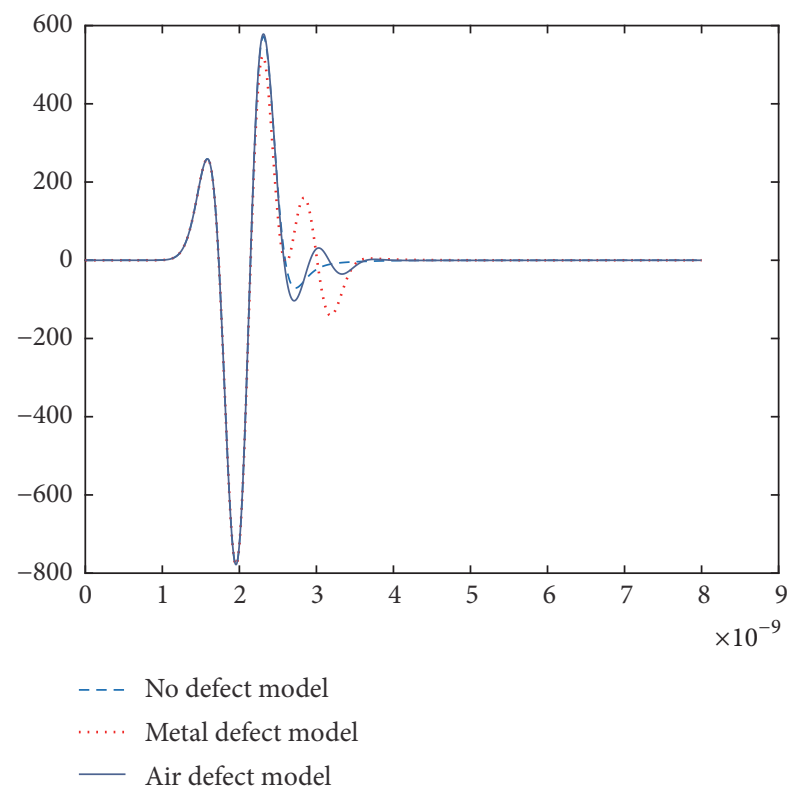

Figure 6: Comparison of A-scan scan data at the same array.

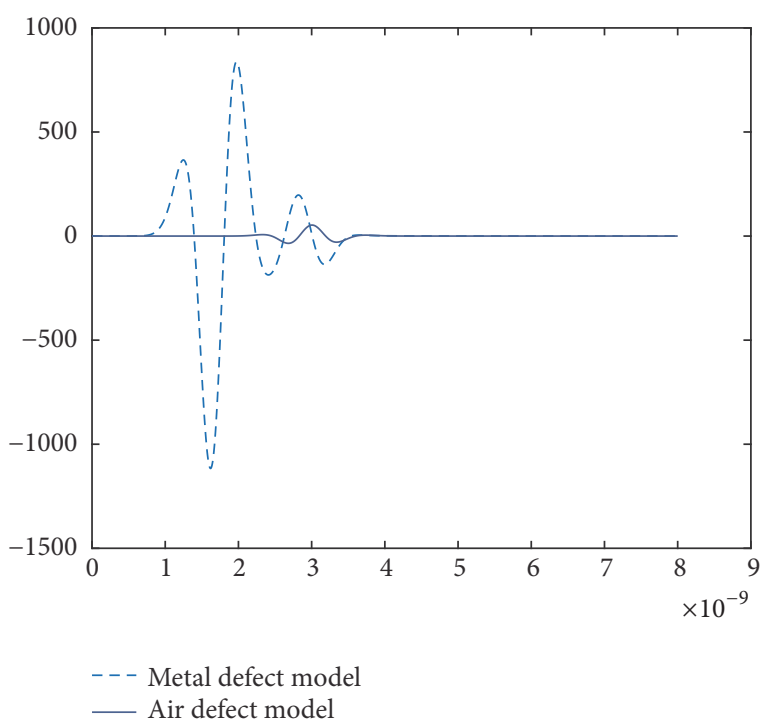

FIGURE 7: Comparison of scattering fields between metal defect model and air defect model.

from the scattering on the circumference of the background medium.

As shown in Figure 7, after subtracting the metal defect model data and the no defect model data, the scattering field data of the metal defect model was obtained. After the air defect model data and the no defect model data were subtracted, the scattering field data of the air defect model was obtained. Comparing the curves of the two models, it can be found that the scattering field data of the two models were not zero in the range of 2.7 to $4 \mathrm{~ns}$, the scattering field intensity of the metal defect in the range of 0 to $2.57 \mathrm{~ns}$ was much larger than the scattering field intensity of the air defect, and the

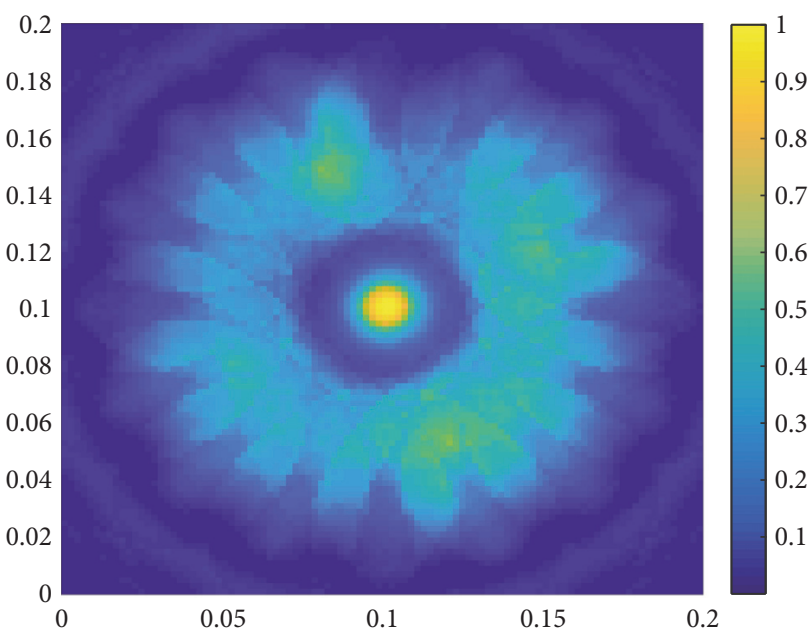

Figure 8: Image of the air defect model after pretreatment.

scattering field of the air defect was similar to the scattering field intensity at 2.57 to $4 \mathrm{~ns}$.

Since we only cared about the scattering field data at the defect and needed to eliminate scattering field data from background media as much as possible, we pre-zero the scattered field data in the range of 0 to $2.57 \mathrm{~ns}$ and 4 to $8 \mathrm{~ns}$ and then made the inversion imaging calculation with the total focusing method. The image of the air defect model after preprocessing is shown in Figure 8.

\subsection{Simulated Imaging of Air Defects in Different Shapes} and Positions. The internal defects of the polymer-modified wood were set to a circular air with a diameter of $2 \mathrm{~cm}$, and the circle center at $(0.1 \mathrm{~m}, 0.05 \mathrm{~m})$ as shown in Figure 9(a). Figure 9(b) shows that the rectangular air gap has a slit length of $0.1 \mathrm{~m}$ and width of $0.02 \mathrm{~m}$. The vertex coordinates are sequentially A $(0.05 \mathrm{~m}, 0.099 \mathrm{~m}), \mathrm{B}(0.05 \mathrm{~m}, 0.101 \mathrm{~m}), \mathrm{C}(0.15 \mathrm{~m}$, $0.101 \mathrm{~m})$, and $\mathrm{D}(0.15 \mathrm{~m}, 0.099 \mathrm{~m})$, by using gprMax software to separate the two models and perform simulation calculations as shown in Figure 9(b). The inversion results are shown in Figures 10(a) and 10(b). The inversion calculation of the total focusing method can accurately detect the shape information of the defects, but the position information of the inversion imaging had a certain error compared with the model. The simulation position of the defect was compared with the model position and it can be found that it rotated about $12.5^{\circ}$ clockwise with the center of the model as the center.

3.5. Multiple Circular Air Defect Simulation Imaging. The internal defects of the polymer-modified wood were set to two circular airs of $0.02 \mathrm{~m}$ in diameter, and the centers at A $(0.05 \mathrm{~m}, 0.1 \mathrm{~m})$ and B $(0.15 \mathrm{~m}, 0.1 \mathrm{~m})$, respectively, as shown in Figure 11(a), with three diameters of $0.02 \mathrm{~m}$. The circular air, centered at $\mathrm{A}(0.7 \mathrm{~m}, 0.7 \mathrm{~m}), \mathrm{B}(0.10 \mathrm{~m}, 0.13 \mathrm{~m})$, and C $(0.13 \mathrm{~m}, 0.07 \mathrm{~m})$ as shown in Figure 11(b), was obtained using the gprMax software to separate the two models' simulation calculation. The inversion results are shown in Figures 12(a) and 12 (b). The inversion calculation of the total focusing 


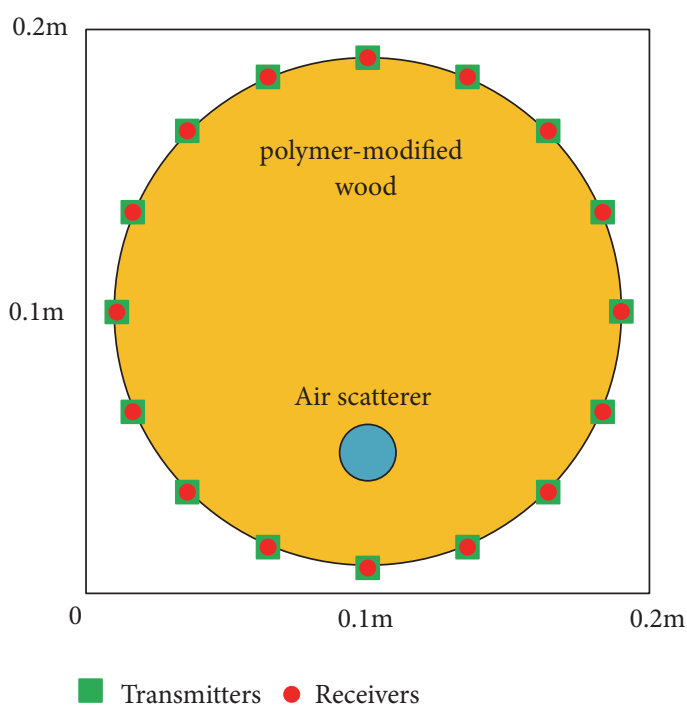

(a) Circular defect model diagram

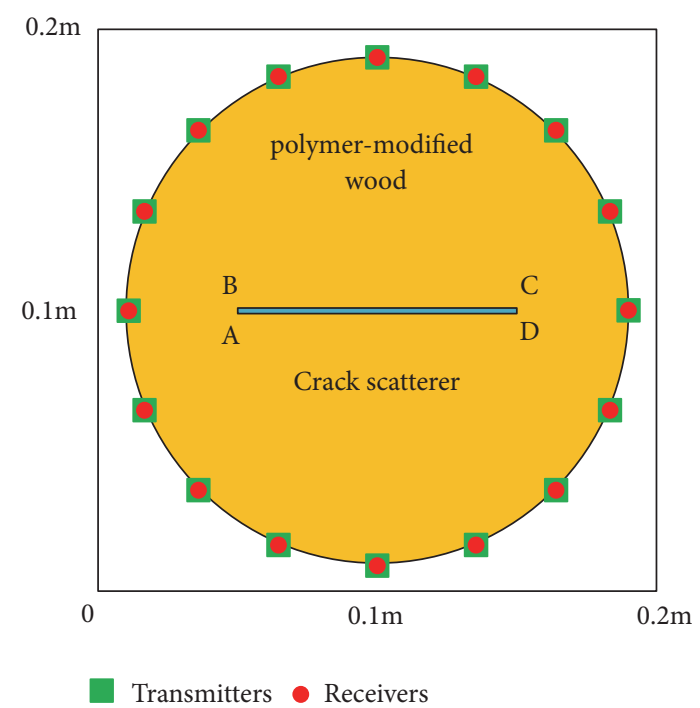

(b) Slot defect model diagram

Figure 9

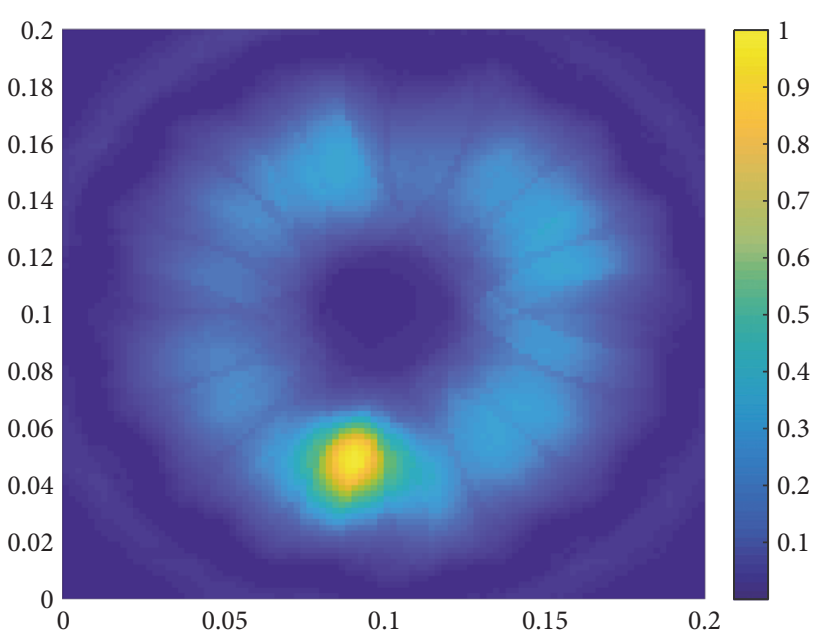

(a) Circular defect inversion imaging

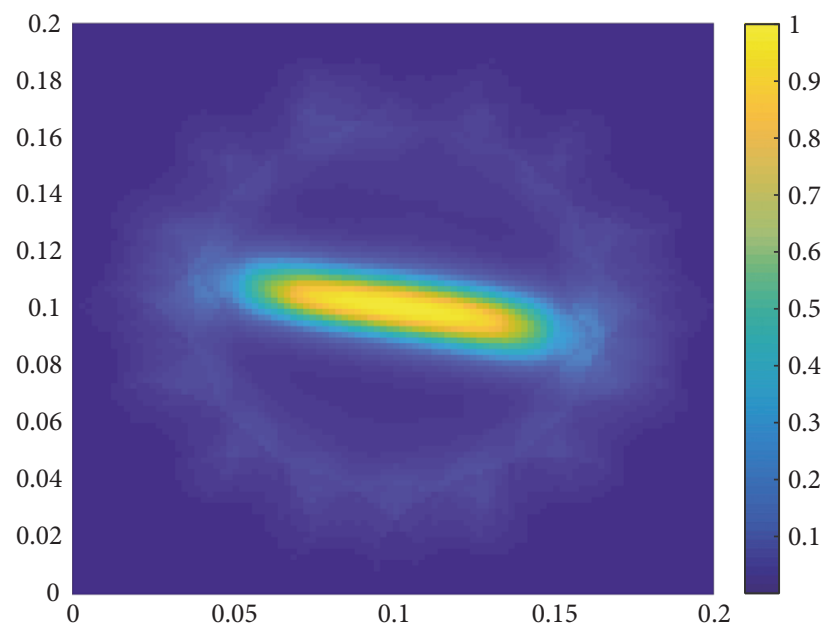

(b) Slot defect inversion imaging

Figure 10

method can detect multiple circular defect information, but the position information had an error of about $12.5^{\circ}$.

3.6. Discussion and Analysis. The total focusing method for electromagnetic wave scattering field data reconstruction that inverted spatial scatterer images from time-domain signals from all combinations of transmit-receive array elements was used in this study. The simulation results showed that the scattering field data could be reconstructed at each sampling point in space using the total focusing method, so that the position where the scattering field value was prominent formed a "bright spot" to achieve the purpose of imaging. The strength of the "bright spot" depended on the scattering intensity of the scatterer on the electromagnetic wave, and the metal scatterer had a better imaging effect than the air scatterer. In order to solve the problem that the air scatterer imaging was not ideal, the direct wave data in the scattered field was preprocessed and then the inversion imaging calculation was performed. The deviation between the simulation imaging results of the gap model and the multicircle model and the position of the scatterer in the model was expected to be solved by increasing the transmitreceive array elements $(N>16)$.

\section{Conclusion}

In this paper, the objective of imaging the internal defects of polymer-modified wood was realized by the forward modeling of electromagnetic wave and the inversion imaging calculation of the total focusing method. By measuring the scattered waves generated at the normal tissue and defect 


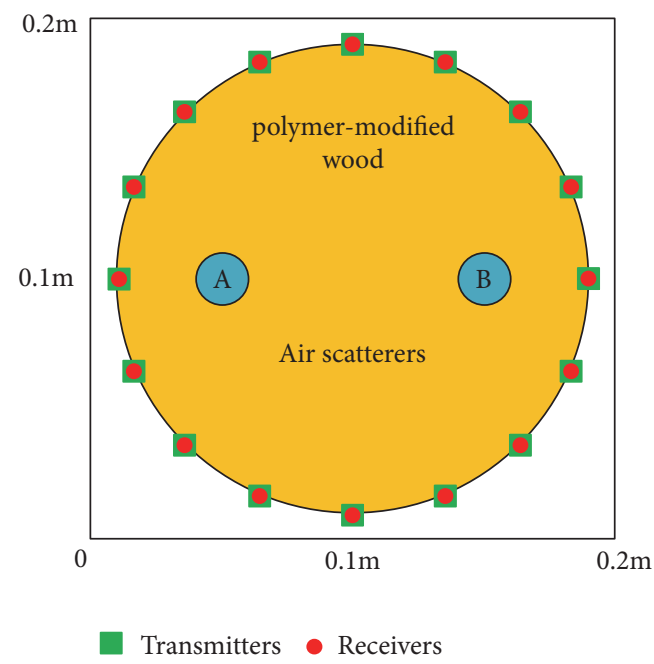

(a) Double-circle defect model diagram

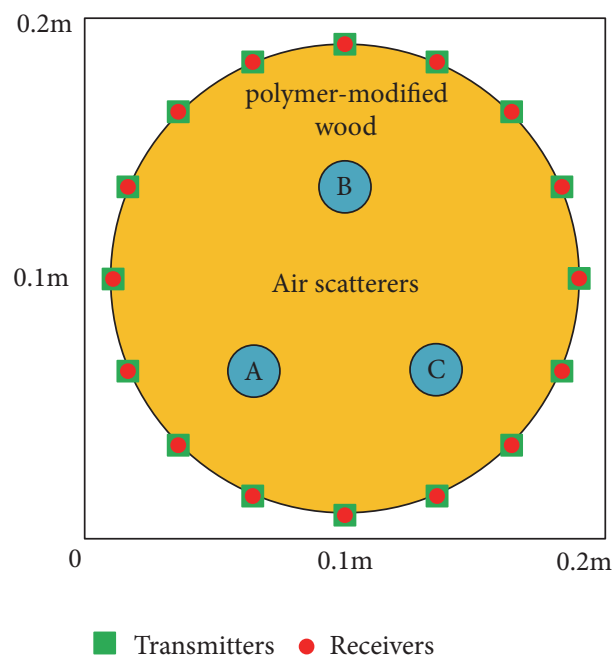

(b) Three-circle defect model diagram

Figure 11

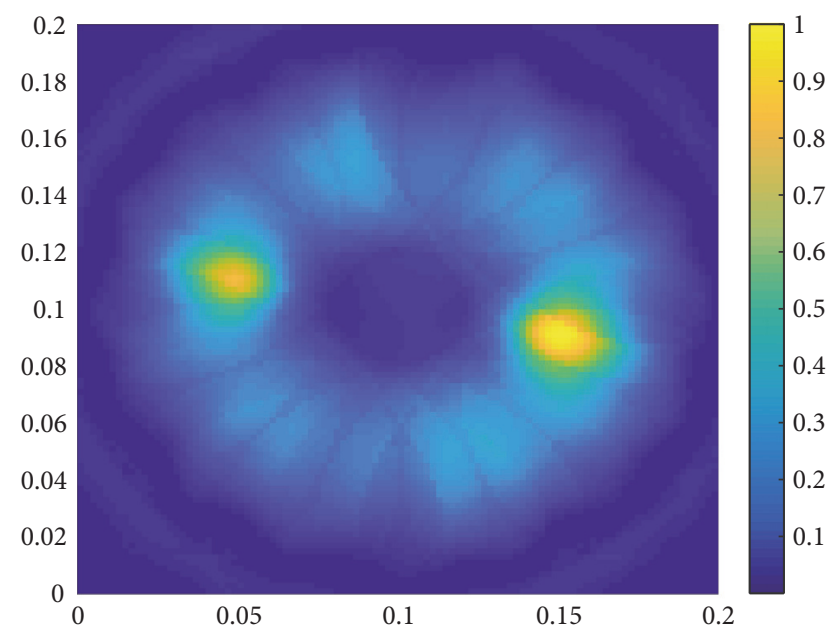

(a) Double-circle defect inversion imaging

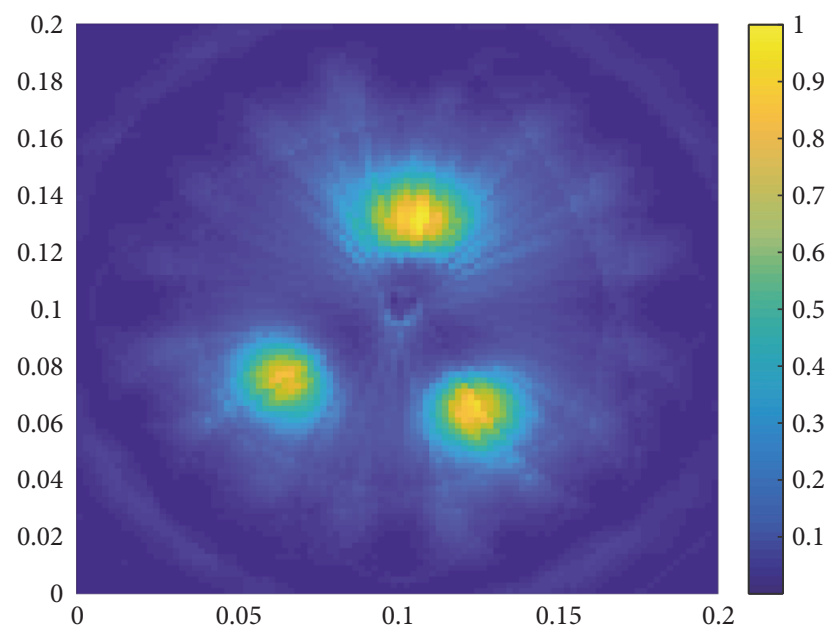

(b) Three-circle defect inversion imaging

Figure 12

interface inside the polymer-modified wood, the type, location, shape, and quantity of internal defects imaging of the polymer-modified wood were recognized according to the differences in the amplitude and speed of electromagnetic wave propagation inside different media. The scheme verified the feasibility of the total focusing method proposed in this paper to realize the inversion imaging of polymer-modified wood internal defects. The experimental results showed that the gprMax software can correctly simulate the polymer material and internal defects. The numerical results can be used for inversion imaging. The scattering intensity of metal defects on electromagnetic waves was large. Accurate defect images can be directly obtained in the inversion calculation of the total focusing method, but for air-based defects, the intensity of the scattered field at the interface was much smaller than the intensity of the direct wave, resulting in a bright spot stronger than the defect at the array element in the inversion image. After the direct wave data at each dot matrix was zeroed, the in-focus image inversion was significantly improved to achieve accurate detection of defective images. In this paper, using electromagnetic wave technology to carry out forward modeling and inversion imaging calculation of polymer-modified wood internal defects, the purpose of nondestructive testing of polymer-modified wood internal defects was realized. The method was simple in operation and fast in imaging calculation. It can provide a basis for the detection of internal defects in other types of polymer products or materials.

\section{Data Availability}

The data used to support the findings of this study are available from the corresponding author upon request. 


\section{Conflicts of Interest}

The authors declare that they have no conflicts of interest.

\section{Acknowledgments}

This work was funded by the National Key Research and Development Program (Grant No. 2017YFD0600101), the China Postdoctoral Science Foundation (Grant No. 2018M640288), and the Heilongjiang Postdoctoral Fund (Grant No. LBH-Z18004).

\section{References}

[1] M. Gaff, M. Babiak, and F. Kacik, "Plasticity properties of thermally modified timber in bending - the effect of chemical changes during modification of European oak and Norway spruce," Composites Part B: Engineering, vol. 165, pp. 613-625, 2019.

[2] X. Wei, D. Tao, X. Sheng, and M. Zhang, "Modification of poplar wood using polyhexahydrotriazine and its effect on hygroscopicity," Journal of Wood Chemistry and Technology, vol. 38, no. 3, pp. 214-223, 2018.

[3] K. Peeters, E. Larnøy, A. Kutnar, and C. A. S. Hill, "An examination of the potential for the use of the Maillard reaction to modify wood," International Wood Products Journal, vol. 9, no. 3, pp. 108-114, 2018.

[4] A. Bastani, S. Adamopoulos, and H. Militz, "Shear strength of furfurylated, N-methylol melamine and thermally modified wood bonded with three conventional adhesives," Wood Material Science and Engineering, vol. 12, no. 4, pp. 236-241, 2017.

[5] M. Ghorbani, A. Nikkhah Shahmirzadi, and S. M. Amininasab, "Physical and Morphological Properties of Combined Treated Wood Polymer Composites by Maleic Anhydride and Methyl Methacrylate," Journal of Wood Chemistry and Technology, vol. 37, no. 6, pp. 443-450, 2017

[6] M. Altgen, S. Adamopoulos, and H. Militz, "Wood defects during industrial-scale production of thermally modified Norway spruce and Scots pine," Wood Material Science and Engineering, vol. 12, no. 1, pp. 14-23, 2017.

[7] J. Laviada, B. Wu, M. T. Ghasr, and R. Zoughi, "Nondestructive evaluation of microwave-penetrable pipes by synthetic aperture imaging enhanced by full-wave field propagation model," IEEE Transactions on Instrumentation and Measurement, vol. 68, no. 4, pp. 1112-1119, 2019.

[8] V. A. Zorin, N. I. Baurova, and E. A. Kosenko, "Detection of defects in components made of dispersion-filled polymeric materials by the method of infrared thermography," Polymer Science - Series D, vol. 10, no. 3, pp. 241-243, 2017.

[9] E. Schneider and C. Boller, "Ultrasonic material characterization and testing of anisotropic components," in Innovative Design and Development Practices in Aerospace and Automotive Engineering, vol. 14, pp. 5-6, 2016.

[10] Z. Li, A. D. Haigh, M. N. Saleh et al., "Detection of impact damage in carbon fiber composites using an electromagnetic sensor," Research in Nondestructive Evaluation, vol. 29, pp. 123142, 2018.

[11] I. P. Shcherbakov, V. I. Vettegren, R. I. Mamalimov, and K. F. Makhmudov, "The influence of stress on electron emission initiated by a shock wave from a heterogeneous material (granite)," Physics of the Solid State, vol. 59, no. 3, pp. 575-577, 2017.
[12] S. Guo, S. Chen, L. Zhang, Y. F. Chen, and K. Yao, "Plastic strain determination with nonlinear ultrasonic waves using in situ integrated piezoelectric ultrasonic transducers," IEEE Transactions on Ultrasonics, Ferroelectrics and Frequency Control, vol. 65, no. 1, pp. 95-101, 2018.

[13] Y.-I. Hwang, J. Park, and H.-J. Kim, "Performance comparison of ultrasonic focusing techniques for phased array ultrasonic inspection of dissimilar metal welds," International Journal of Precision Engineering and Manufacturing, vol. 20, pp. 525-534, 2019.

[14] W. Jiangfei, Y. Lihua, Z. Zhengguang, and Y. Mingyuan, "Accurate detection of a defective area by adopting a divide and conquer strategy in infrared thermal imaging measurement," Journal of the Korean Physical Society, vol. 73, pp. 1644-1649, 2018.

[15] N.-S. Kwak, J.-Y. Kim, and J.-C. Gao, "Detection of small-flaw in carbon brake disc (C-C) using air-coupled ultrasonic C-scan technique," International Journal of Precision Engineering and Manufacturing, vol. 18, pp. 987-994, 2017.

[16] R. B. Roy, A. Ghosh, S. Bhattacharyya et al., "Weld defect identification in friction stir welding through optimized wavelet transformation of signals and validation through X-ray microCT scan," The International Journal of Advanced Manufacturing Technology, vol. 99, pp. 623-633, 2018.

[17] Y. Jiang and S. Yin, "Recursive total principle component regression based fault detection and its application to vehicular cyberphysical systems," IEEE Transactions on Industrial Informatics, vol. 14, no. 4, pp. 1415-1423, 2018.

[18] A. Muller, B. Robertson-Welsh, P. Gaydecki, M. Gresil, and C. Soutis, "Structural health monitoring using lamb wave reflections and total focusing method for image reconstruction," Applied Composite Materials, vol. 24, no. 2, pp. 553-573, 2017.

[19] Holmes C., B. W. Drinkwater, and P. D. Wilcox, "Postprocessing of the full matrix of ultrasonic transmit-receive array data for non-destructive evaluation," NDT\&E International, vol. 38, pp. 701-711, 2005.

[20] J. zhang, B. W. Drinkwater, and D. Paul, "Defect detection using ultrasonic arrays: the multi-mode total focusing method," NDT and E International, vol. 43, pp. 123-133, 2010.

[21] H. Zhou, L. Sun, Y. Yang et al., "Reduction of electric field strength by two species of trees under power transmission lines," Journal of Forestry Research, vol. 29, no. 5, pp. 1415-1422, 2018.

[22] H. Zhou, L. Ma, L. Sun, H. Zhou, D. Liu, and L. Cai, "Impact moderation of power transmission lines on the environment by the shielding of trees," BioResources, vol. 13, no. 4, pp. 82398250, 2018. 


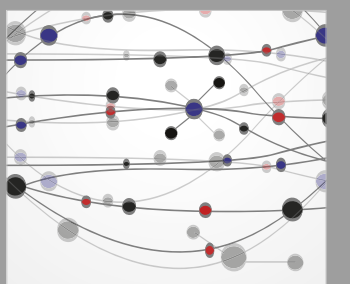

The Scientific World Journal
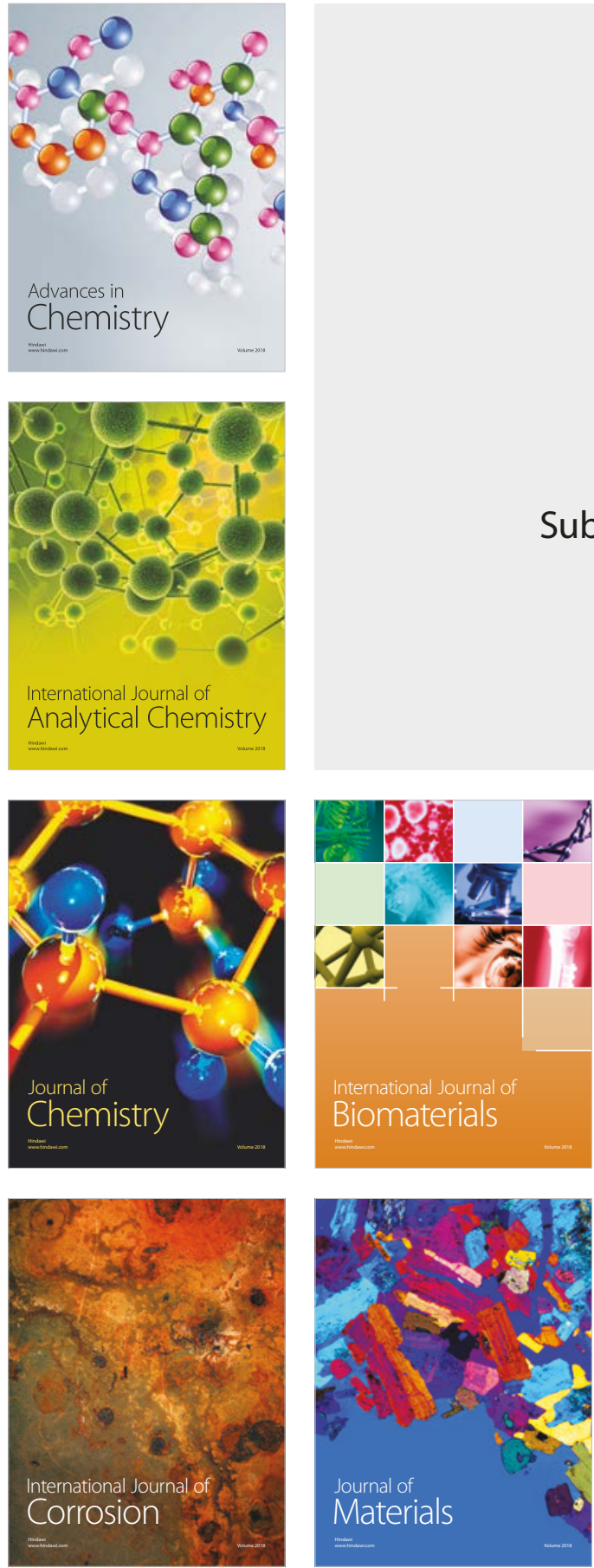

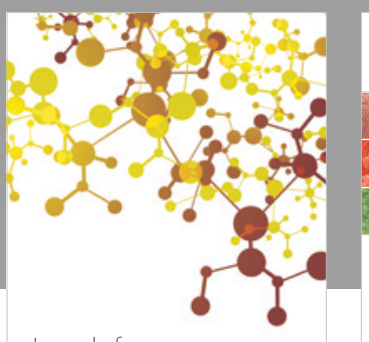

Journal of

Applied Chemistry
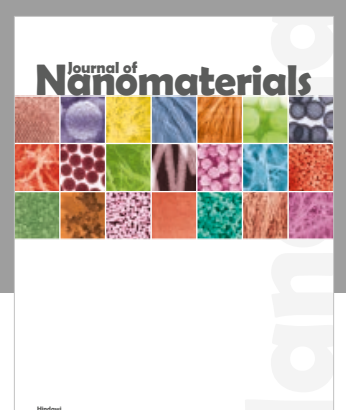

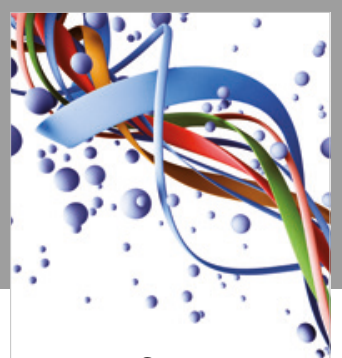

Scientifica

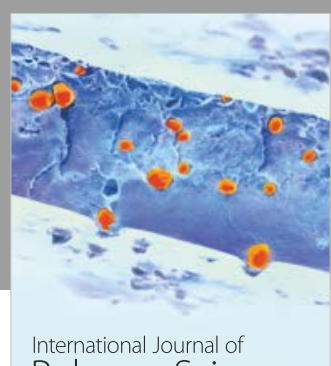

Polymer Science

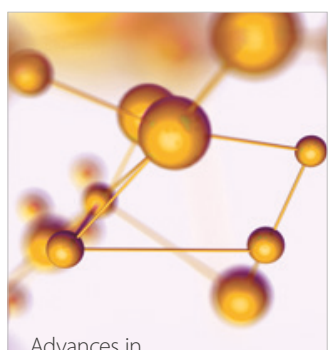

Physical Chemistry
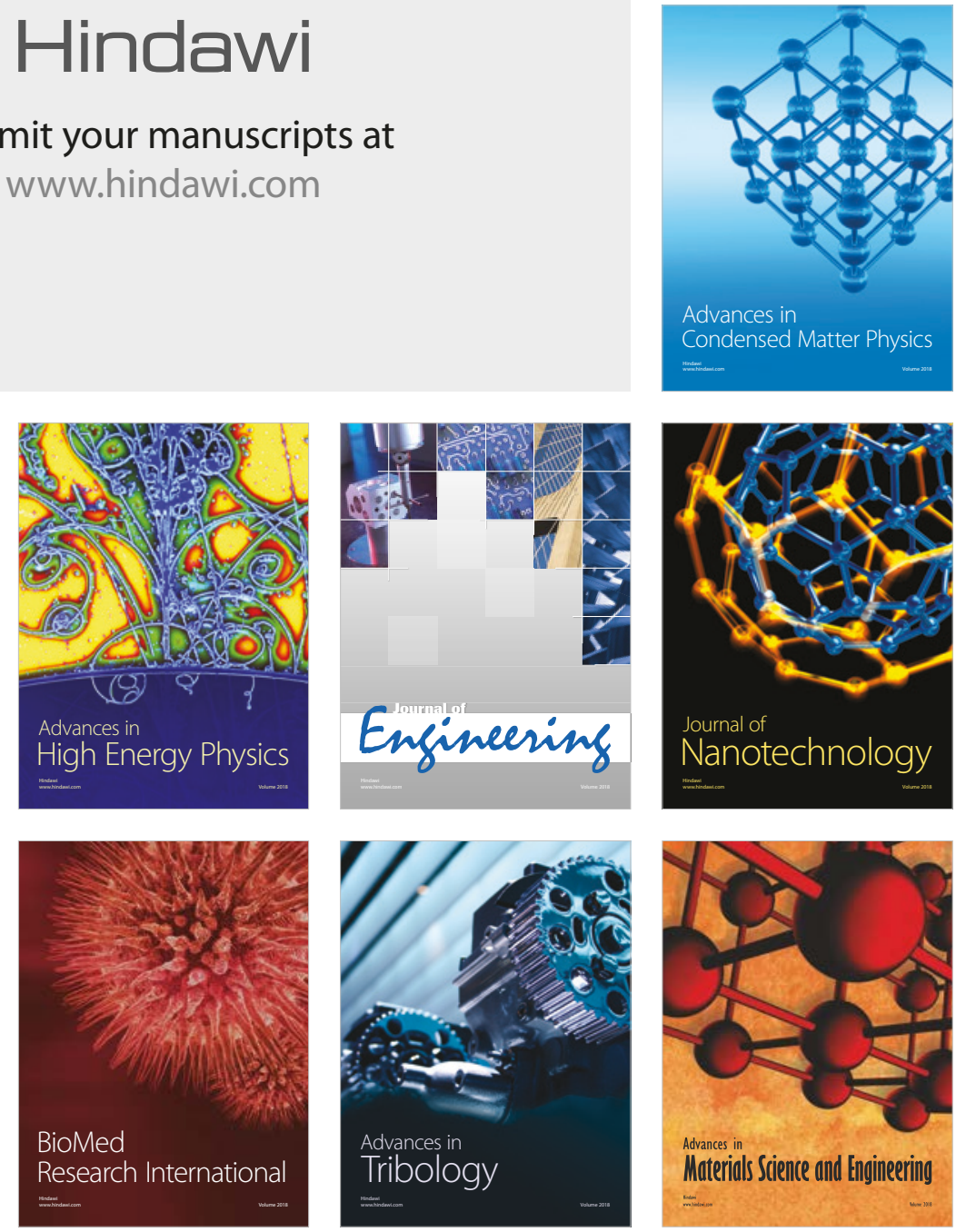